\title{
The Distribution of Variable Stars in the Galaxy
}

\section{PLAUT (Groningen)}

At first, I have to thank Dr. STROHMEIER sincerely for his invitation to the fifth colloquium on variable stars.

After SHAPLEY recognized the variable stars as a very useful tool in obtaining the distribution of stars in the galactic system. Dr. HOFFMEISTER was one of the first to investigate the distribution of variable stars by the aid of series of photographic plates of selected regions in the Galaxy. At his observatory at Sonneberg he and his collaborators took many plates which were searched for variables and on which the brightness of these stars was estimated and their distribution studied. The results of these studies are published in a long series of papers in the Sonneberg publications.

The reason why variable stars are so suitable for the determination of the stellar distribution is obvious: they can be recognized as such easily, at least much easier than other types of stars, and, at the same time, to much farther distances.

Several observational programs in this direction were carried out. The Sonneberg project was mentioned already; others are SHAPLEY's extensive plan at Harvard Observatory, the Leiden-Johannesburg series, and, recently, KINMAN's investigations at Lick Observatory and the Palomar-Groningen variable-star survey. In the following I shall mainly concern with the last one.

The first problem of such an investigation is the discovering of the variables. Discovery methods are well known. They are based on comparisons of plates of a region taken at various times. After the variables have been found the light curves or elements have to be derived. We then consider each class of objects separately.

In order to get the spatial distribution we need the distances of the individual stars. These can be obtained by the well-known relation

$$
M=m-5 \log r+5-\text { absorption }
$$

This means that we have to know:

1. The apparent magnitude $\mathrm{m}$, let us say $\mathrm{m}_{\mathrm{pg}}$ or $\mathrm{B}$, down to about 20 magn. That means we need a sequence of standard stars, if possible, at several regions in the field in order to eliminate the field correction.

2. The absolute magnitude of the type of variables considered as a function of some physical parameter, for instance; for RR Lyrae stars as a function of the metal-content parameter $\Delta \mathrm{S}$, defined as $10[\mathrm{Sp}(\mathrm{H})-\mathrm{Sp}(\mathrm{Ca})]$ by PRESTON. This quantity $\Delta \mathrm{S}$ can be replaced by the position in a period-amplitude diagram; for long-period variables as a function of the period, at least as long as no other physically relevant parameter is found. - Most other types of variables are not numerous enough to be considered in this respect. Since in the vicinity of the Sun, classical cepheids of type I can be discussed now the period-luminosityradius relation seems to be known rather reliably, especially from the recent investigations by SANDAGE and TAMMANN.

3. The photographic interstellar absorption $=4 E_{B}-V=4\left[(B-V)_{0}-(B-V)\right]$; consequently we have to know the intrinsic colour $(B-V)_{0}$ and we have to observe $V$, in addition to $B$, and, hence, we need the magnitude scale in $V$, too, down to about $20 \mathrm{~m}$.

4. The so-called completeness factor, because we know that not all variables stars were discovered during the blinking. This completeness factor can be split in two factors, the first arising from the fact that the chance of discovering a magnitude difference $\Delta \mathrm{m}$ between the two plates of a blinked pair is a function of $\Delta \mathrm{m}$ and of $\mathrm{m}$, depending on the observer and on the plates; the second factor depending on the frequency distribution of the $\Delta \mathrm{m}$ 's, which can be derived from the shape of the light curve. 


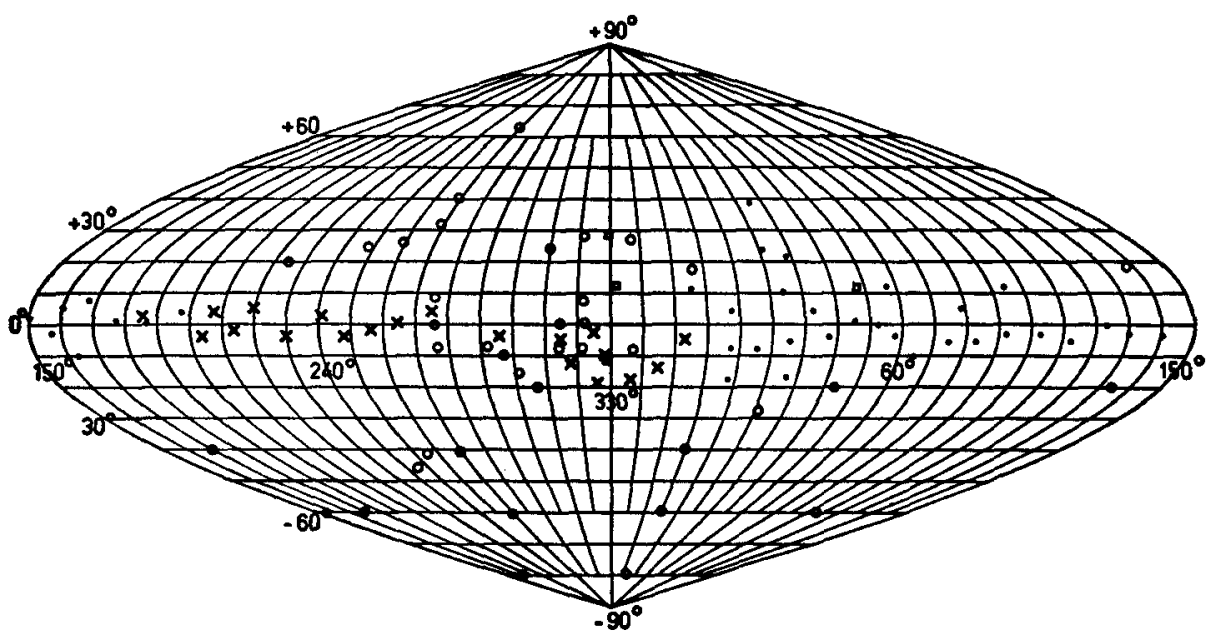

Fig. 1: Regions observed for variable stars: - Sonneberg fields, $\circ$ Harvard fields, $\times$ LeidenJohannesburg fields, $\square$ Palomar fields.

These remarks show the principle. In practice, however, the procedure is full of traps. The absolute magnitudes are not yet accurately known. The troubles on the MV of RR Lyraes are well known: CLUBE, recently suggested a correction of about +0.5 mag., bringing $\left\langle M_{V}\right\rangle$ to about +1 m. Absolute magnitudes of long-period variables are still more uncertain; I may mention only a recent investigation by BARNES on $M_{I}$ of these stars, and the work by CLAYTON and FEAST. Better and more proper motions and radial velocities are needed. The recently derived new plate constants for the old Carte du Ciel plates certainly will be very valuable in this respect.

A further problem ist the interstellar absorption, especially at low galactic latitudes. To show this, let us consider the results of the Palomar survey in more details:

Three fields at about $\mathrm{l}=0^{\circ}$ and $\mathrm{b}=+29^{\circ},+12^{\circ}$ and $-10^{\circ}$ were investigated.

In Fig. 2 lines of equal $\Delta \mathrm{S}=$ metal content are approximately parallel to the line drawn, which represents $\Delta \mathrm{S}=5$. We see that at high latitudes low-metal content stars are more frequent than at low latitudes. The Draco system seems to contain stars of very low metal content.

Figures 3,4 , and 5 show plots of $\langle\mathrm{B}-\mathrm{V}\rangle$ as a function of the galactic latitude. Fig. 3 and 4 concern field 2, the RR Lyraes and the long-period variables. Figure 5 concerns the RR Lyraes in field 3. The upper diagrams show individual stars. We see, that in field 2 the RR Lyraes show an interstellar absorption, which is much larger than the cosecant law at $1=+12^{\circ}$. The other plots approximately agree with a cosecant law.

The following three figures show the results, the abscissae are the distances and the ordinates the logarithms of the densities expressed in RR Lyrae stars per cubic kiloparsec.

In Figure 6: crosses and dashed lines according to KINMAN, $b=+29^{\circ}$; dots according to Palomar field 1 , also at $b=+29^{\circ}$.

There is good agreement between both determinations. 


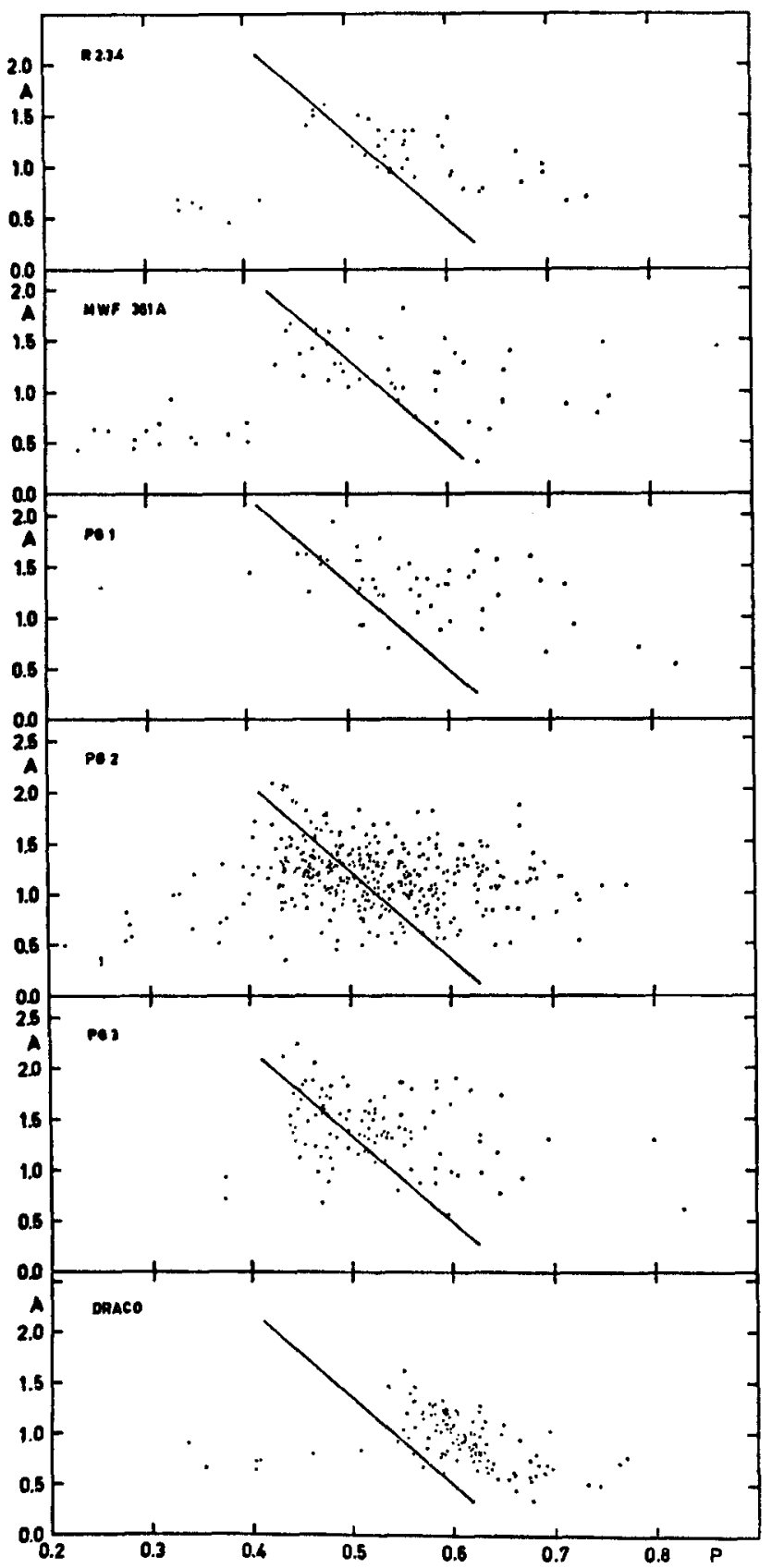

Fig. 2: Period-amplitude diagrams

$\mathrm{R} 2,3,4=$ KINMAN's region near the north galactic pole; MWF $361=$ KINMAN's field at $1=0^{\circ}, b=+29^{\circ}$;

PG $1,2,3=$ Palomar fields at $1=0^{\circ}, b=+29^{\circ},+12^{\circ}$ and $-10^{\circ}$;

Draco = Draco dwarf galaxy according to BAADE and SWOPE. 


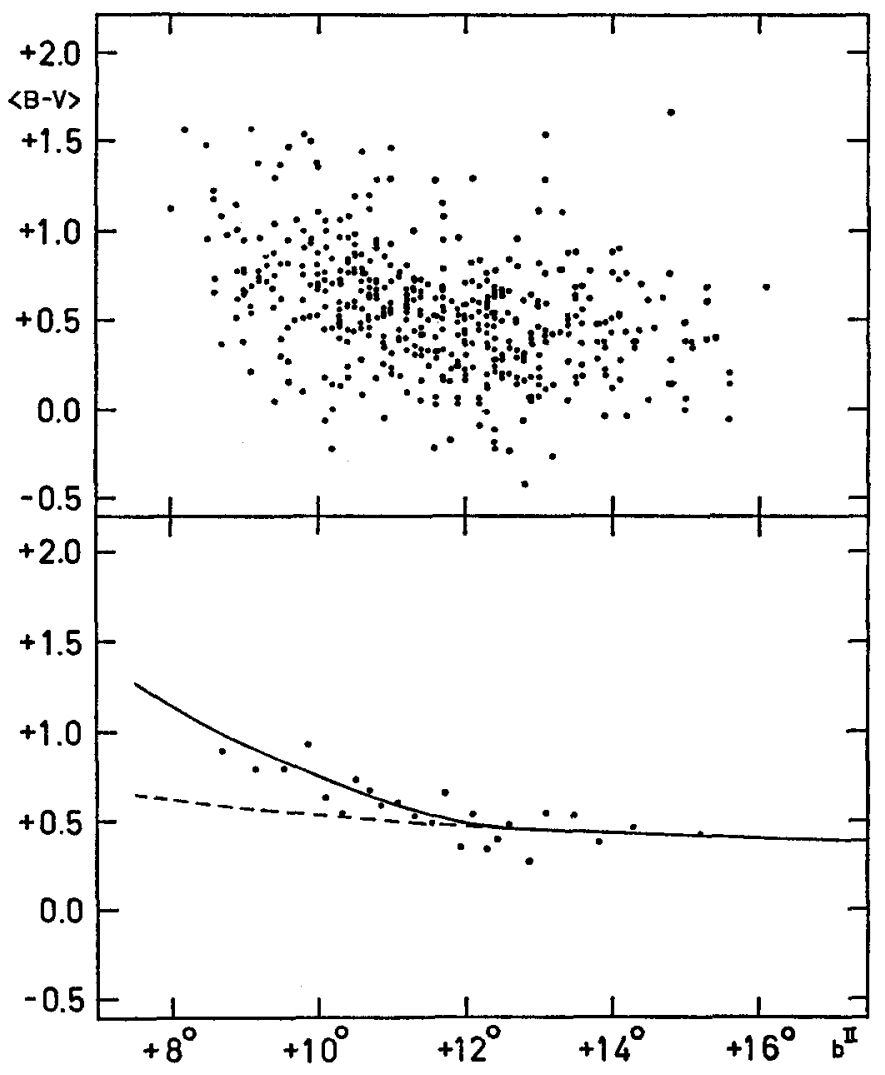

Fig. 3

Figure 7 concerns field 2 at $b=+12^{\circ}$; the field is divided in two parts: $\bullet$ for $b<+12 \%$, $x$ for $b \geqq+12.0^{\circ}$. In the upper plot the absorption is adopted according to the observed colour indices (see Figure 3), in the lower one according to the cosecant law. The first assumption, however, leads to the implausible result that the maximum density is reached for the region $b<+12 \%$ at a smaller distance than for $b \geqq+12 \%$. The second assumption yields a more plausible picture of the density distribution but the assumed lower absorption contradicts the observed colour indices.

Figure 8 gives the result for field 3 at $b=-10^{\circ}$; the photographic absorption is assumed to be equal to $4(+0.03+0.029 \operatorname{cosec} b)$.

Let us now project the densities on a plane perpendicular to the galactic plane through the sun and the galactic centre assumed at a distnce of $10 \mathrm{kpc}$ (Figure 9). Values in parantheses are from KINMAN, WIRTANEN and JANES. Values from positions further than the galactic rotational axis are reflected around this axis. These numbers show that the "reflected" densities are lower than the nearer ones, which probably means that the completeness factors for fainter magnitudes (= larger distances) are too low. 


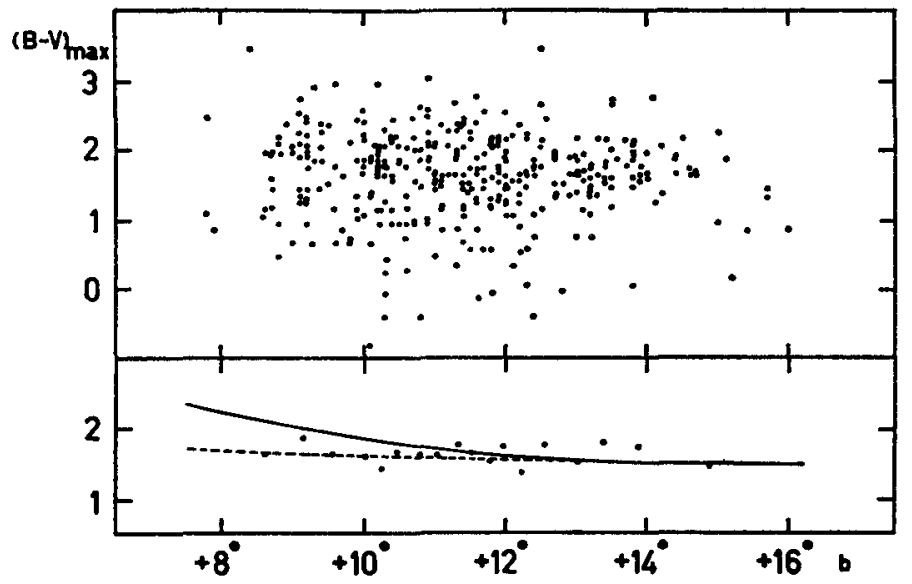

Fig. 4

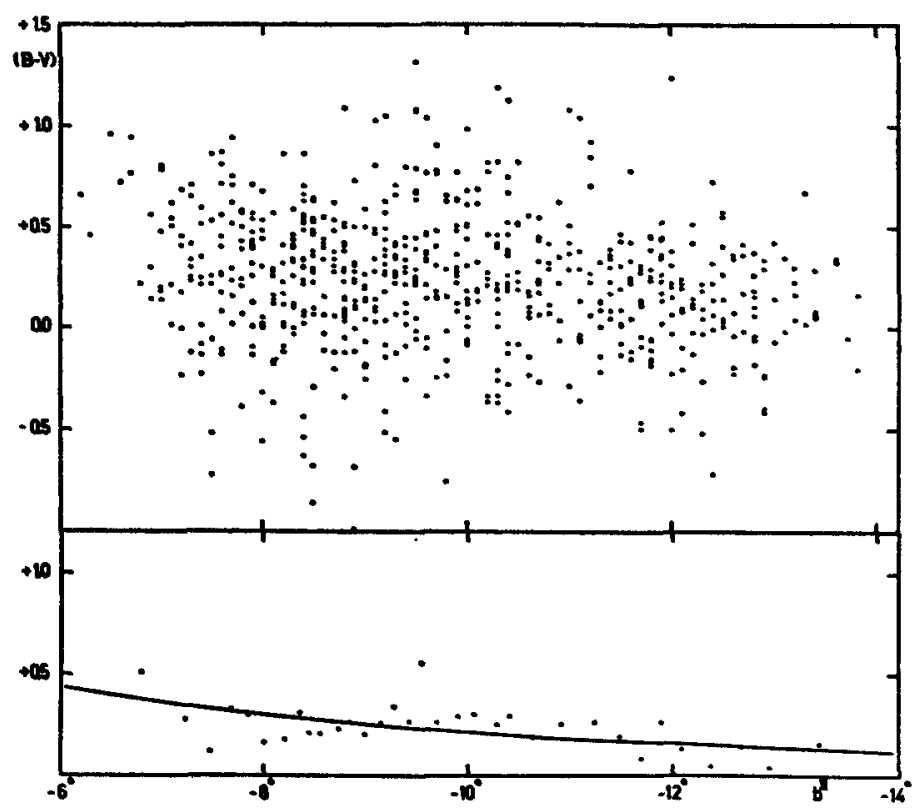

Fig. 5 


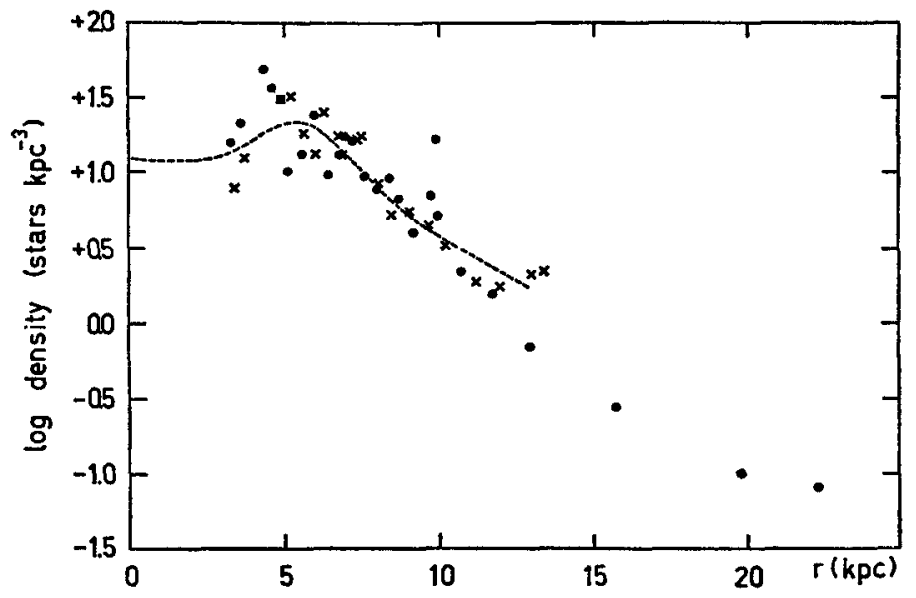

Fig. 6

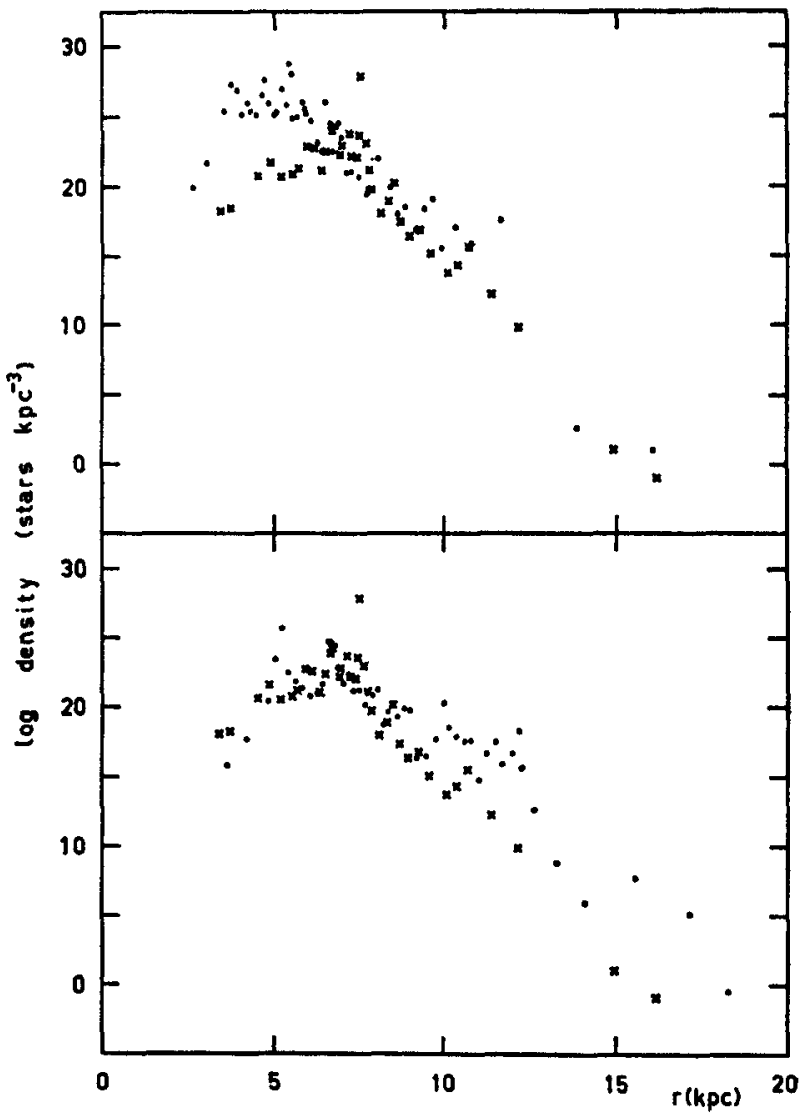

Fig. 7 


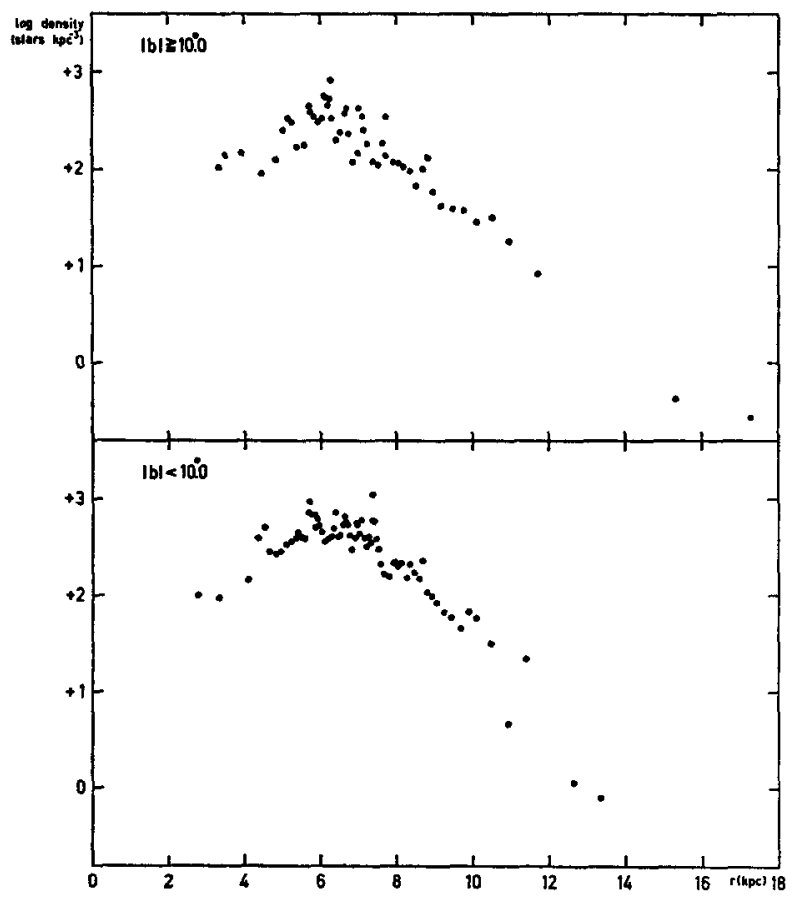

Fig. 8

A similar conclusion may be made from the next figure, no. 10, which gives the densities of the RR Lyraes as a function of the distance $|z|$ from the galactic plane for several values of the distance $R$ from the galactic rotational axis. Dots are derived from stars on the solar side, circles from those on the farther side of the rotational axis. The solid and dashed lines represent the densities derived by WHITE and by PEREK, respectively.

The next two figures, nos. 11 and 12, give the corresponding results for the long-period variables.

At least, let us look at the maximum densities in the plots of the densities against distance. The distances of these maxima correspond to the tangential points of the line of sight on the surfaces of equal density, which can be assumed to be spheroids. As long as $|b|$ is small these distances are nearly equal to the distance to the galactic centre. However, we see from the following table

\begin{tabular}{cll}
\hline$|\mathrm{b}|$ & field & $\mathrm{r}$ (max.) \\
\hline $8^{\circ}$ & $3 \mathrm{l}$ & $6 \mathrm{kpc}$ \\
10 & $2 \mathrm{l}$ & 6 \\
12 & $3 \mathrm{~h}$ & 6 \\
14 & $2 \mathrm{~h}$ & 7 \\
29 & 1 & 5
\end{tabular}

that the distances are much smaller than the usually adopted value of $10 \mathrm{kpc}$. However, I want to emphasize that several reasons exist to be utmost carefully considered before adopting the conclusion that the distance to the centre really is smaller than $10 \mathrm{kpc}$ : 


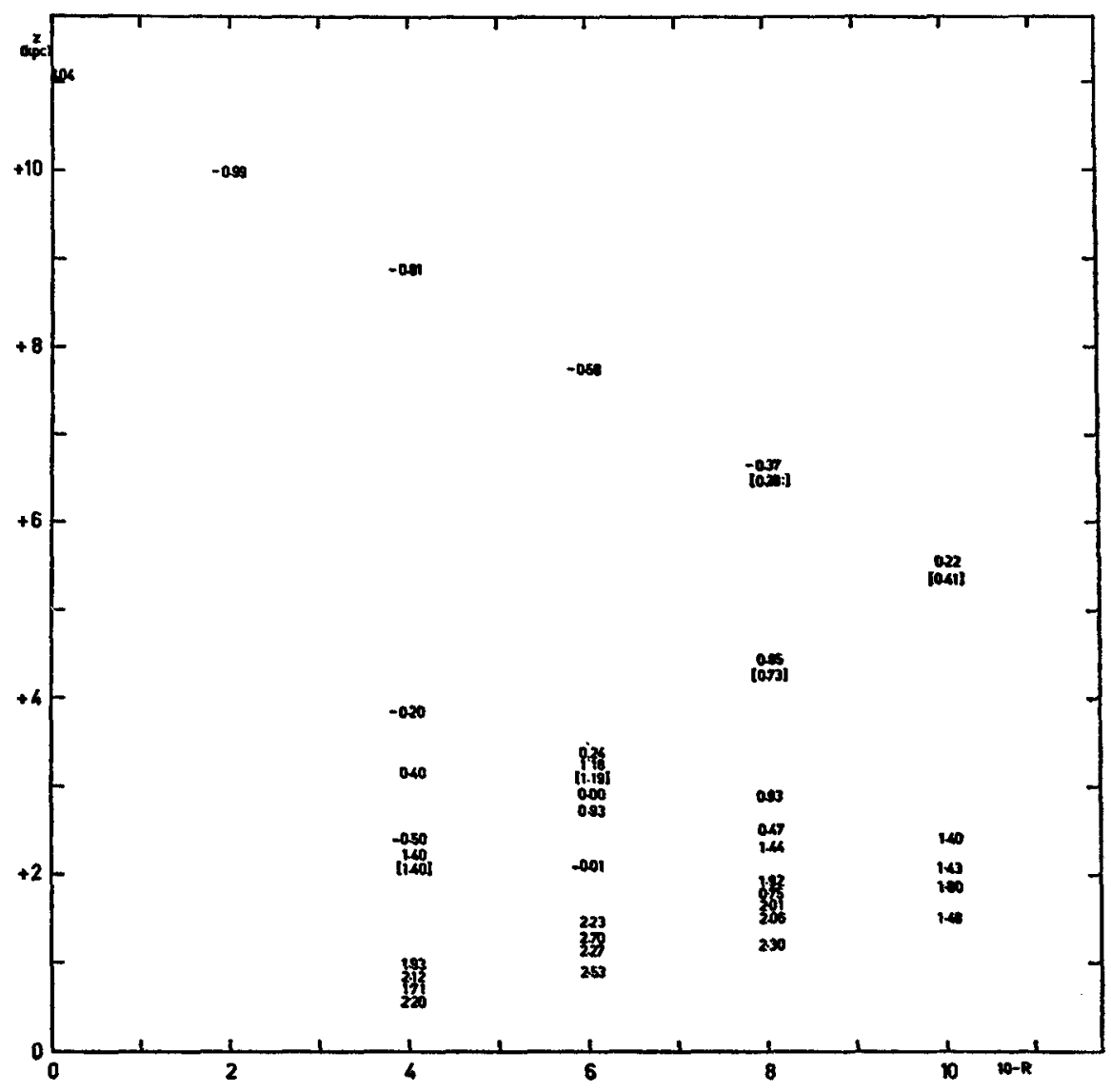

Fig. 9

1. No corrections were applied for the dispersion in $\mathrm{MV}$ and in the observations;

2. The adopted value of $\left\langle\mathrm{M}_{\mathrm{pg}}\right\rangle=+1.0$, may be too faint. However, CLUBE recently suggested even a fainter value. A change of -0.5 in $\left\langle M_{p g}\right\rangle$ will result in multiplying all distances by a factor 1.26 .

3. Zero point and magnitude scale need a redetermination. Down to $m=16.0$ new, not yet reduced, observations from E.S.O. are now available.

4. The interstellar absorption is not well known. The present observations suggest an absorption higher than used in deriving the densities. In addition, there are only very few galaxies visible in the regions near $b= \pm 12^{\circ}$. But a higher absorption will decrease the distance!

5. The completeness factors should be re-examined.

$$
\text { Reference: }
$$

Astronomy and Astrophysics 8, 341, 1970. 

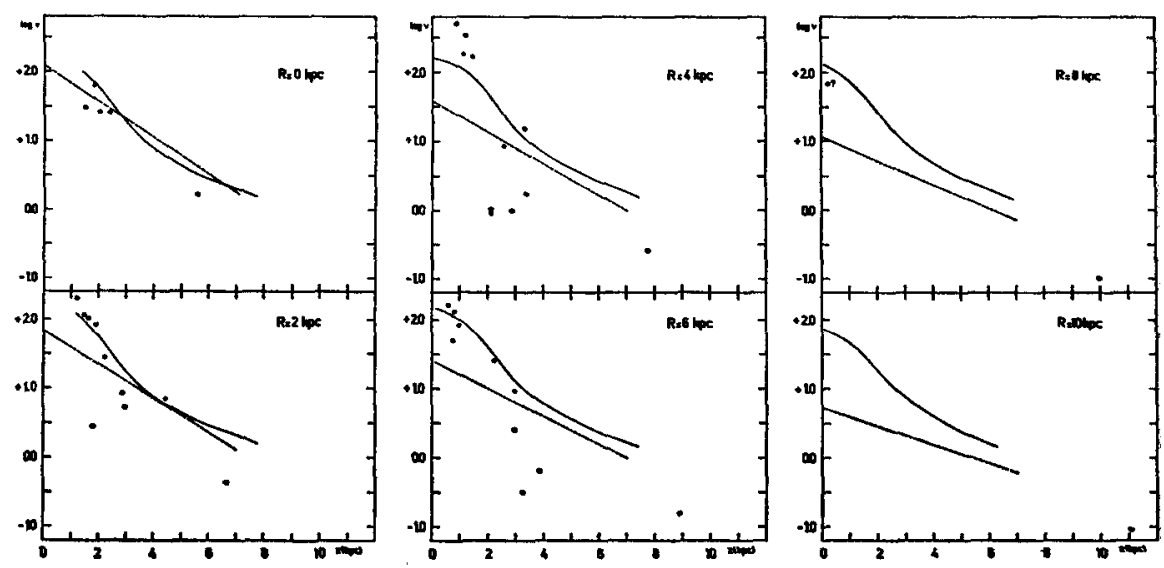

Fig. 10

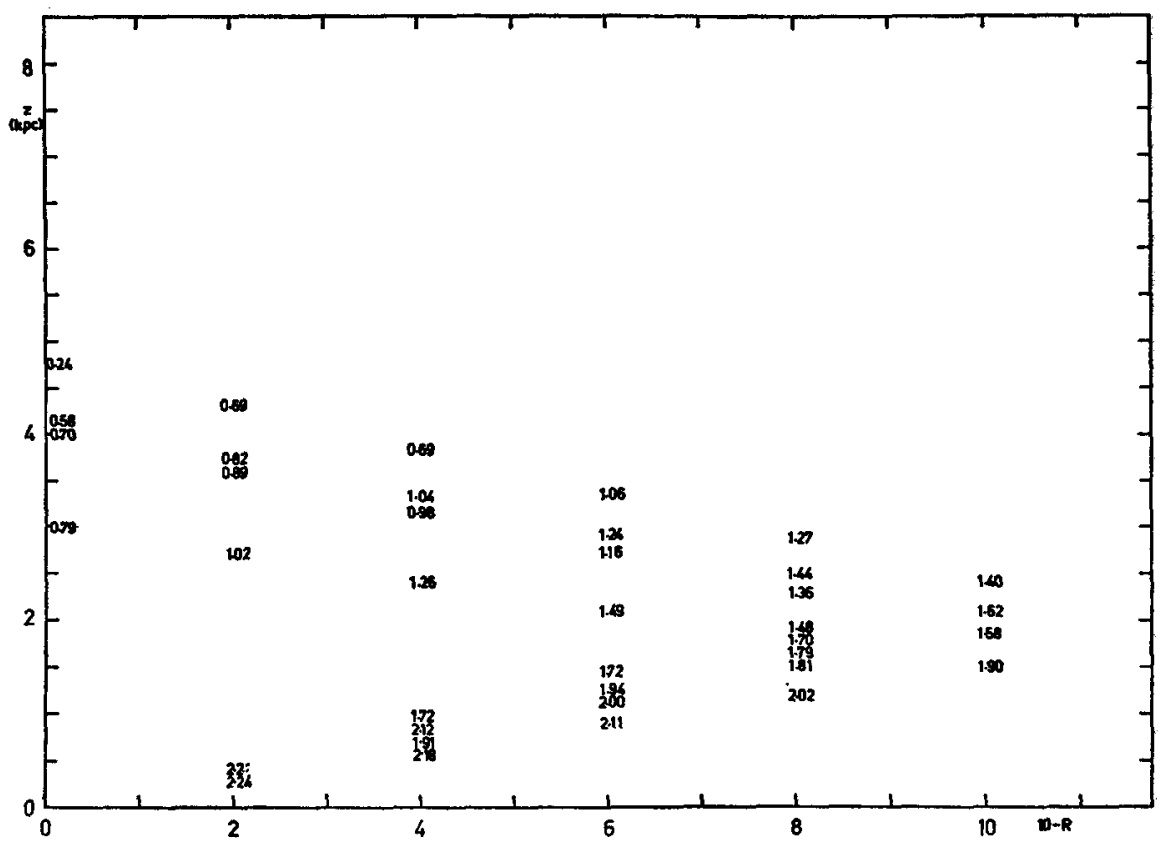

Fig. 11 


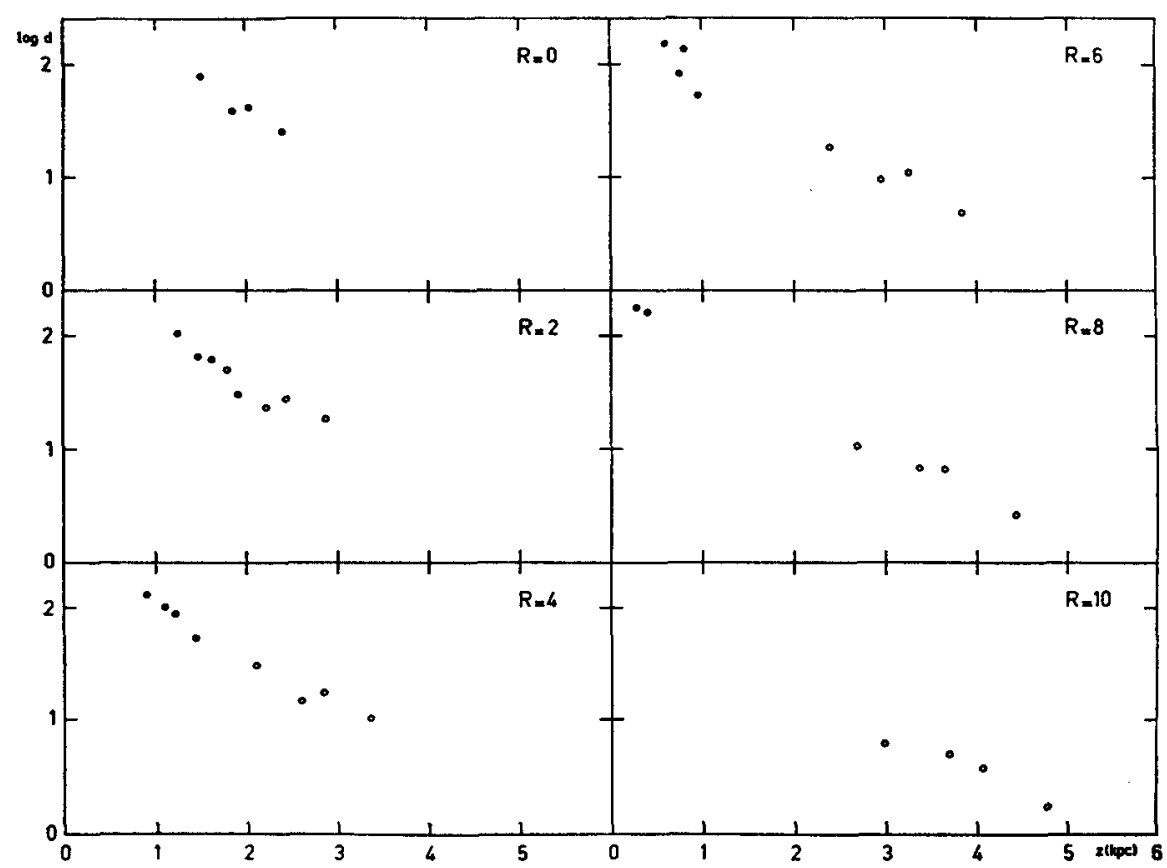

Fig. 12 


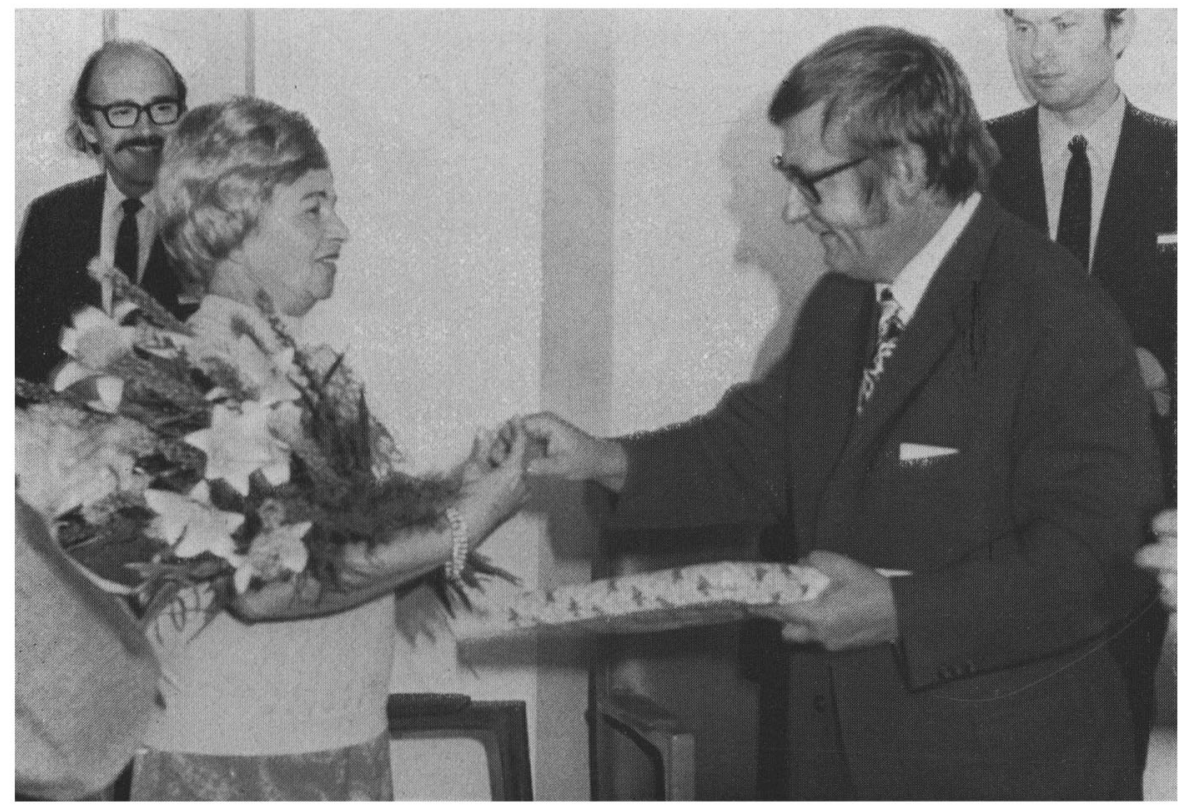

Baker

Gerneth

Kippenhahn

Schöffel

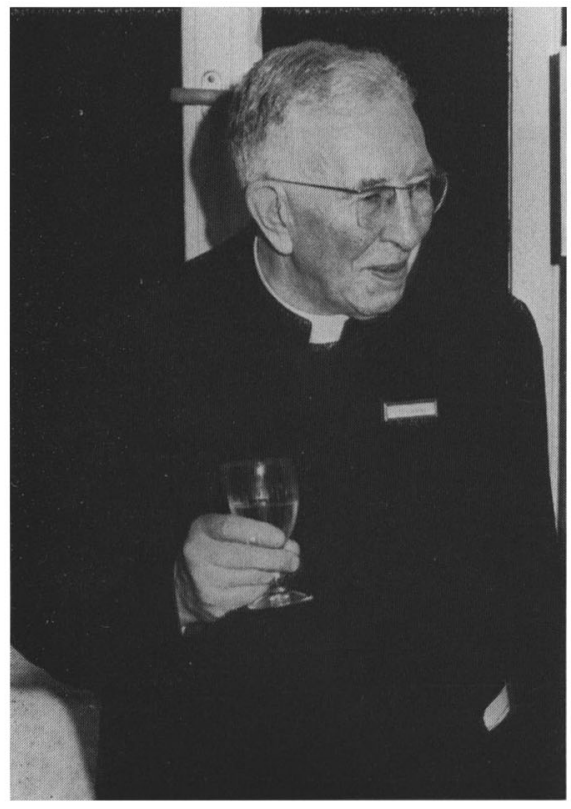

O'Connell

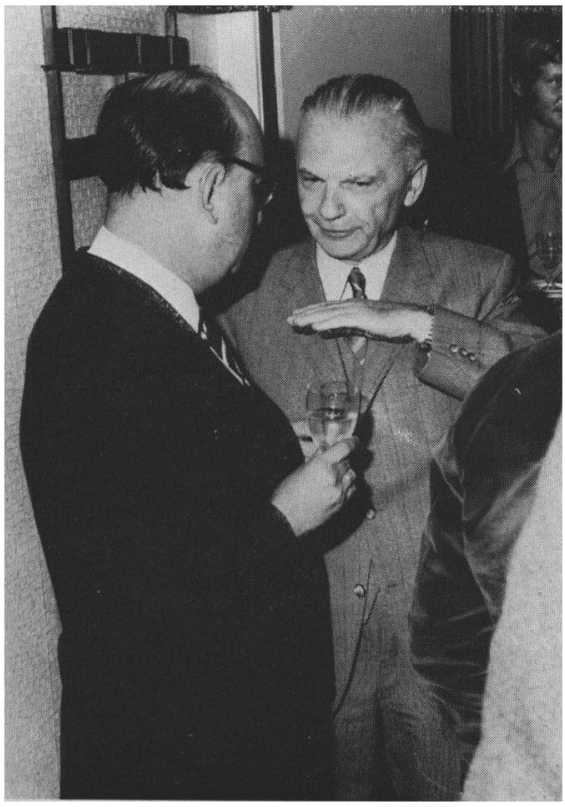

Prorektor Ilschner Strohmeier 


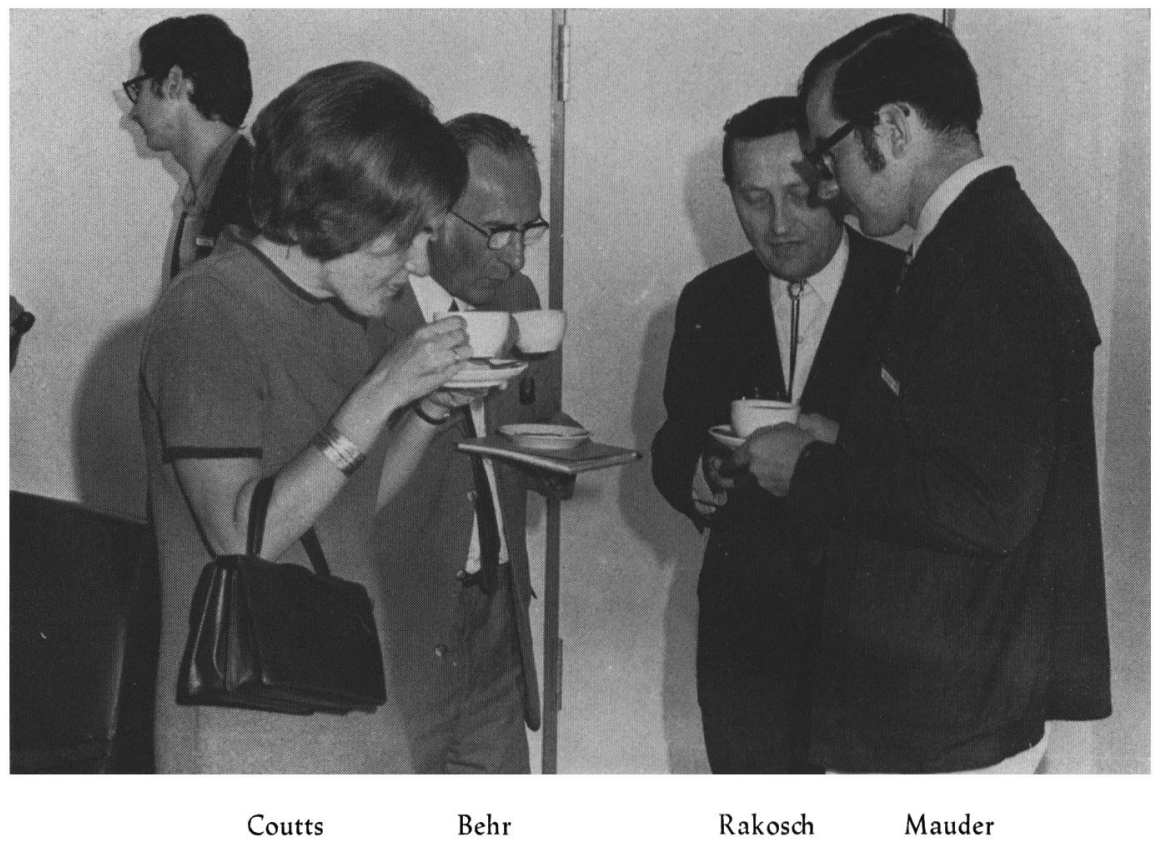

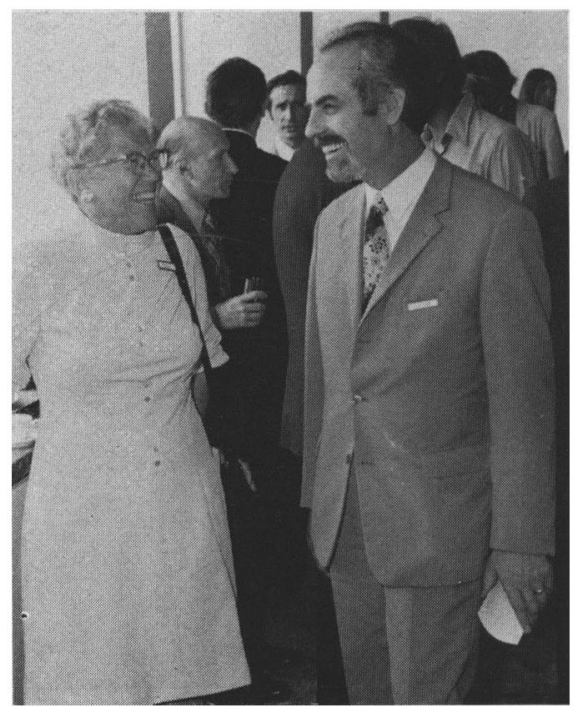

Mayall

Geyer

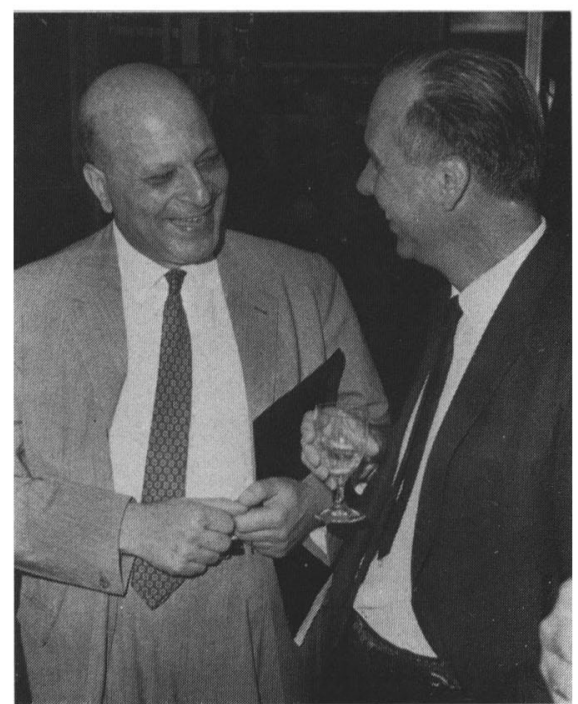

Sahade

Walker 


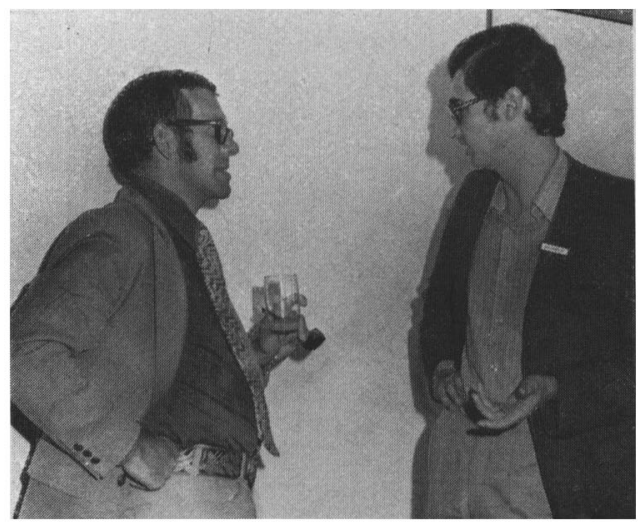

Fernie

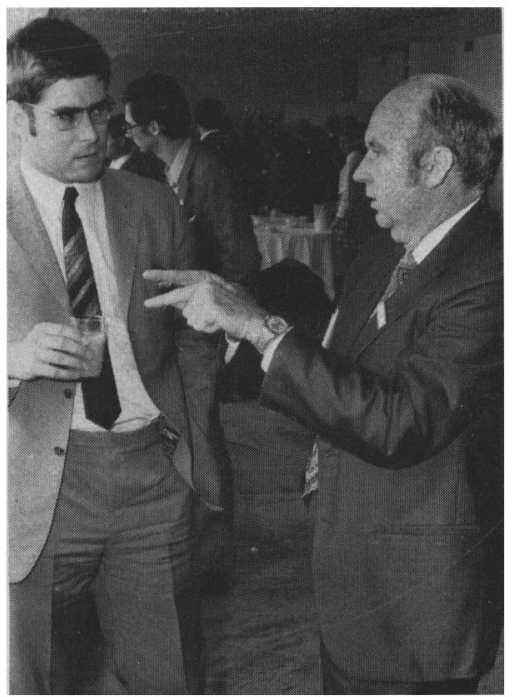

Quester

Wood

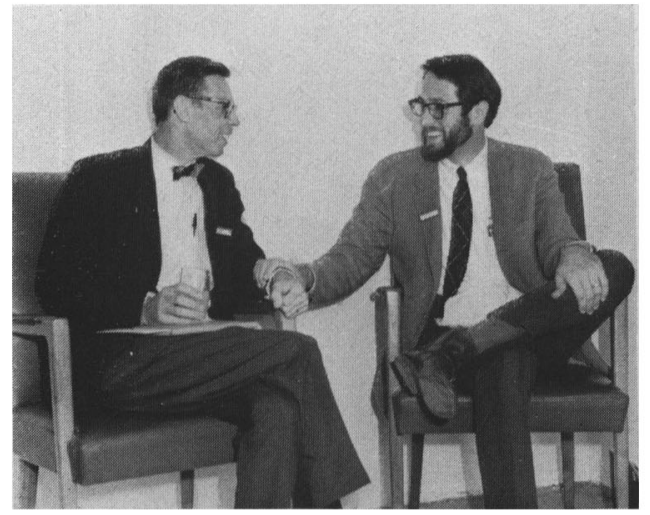

Koch

Hall

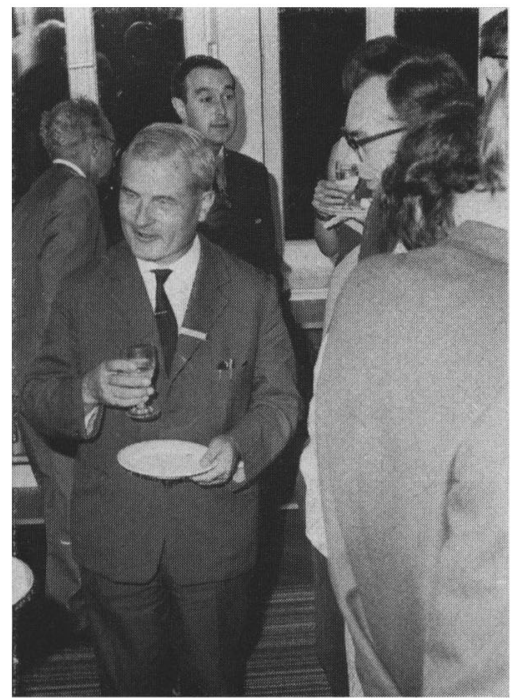

Bertiau

Ciatti 


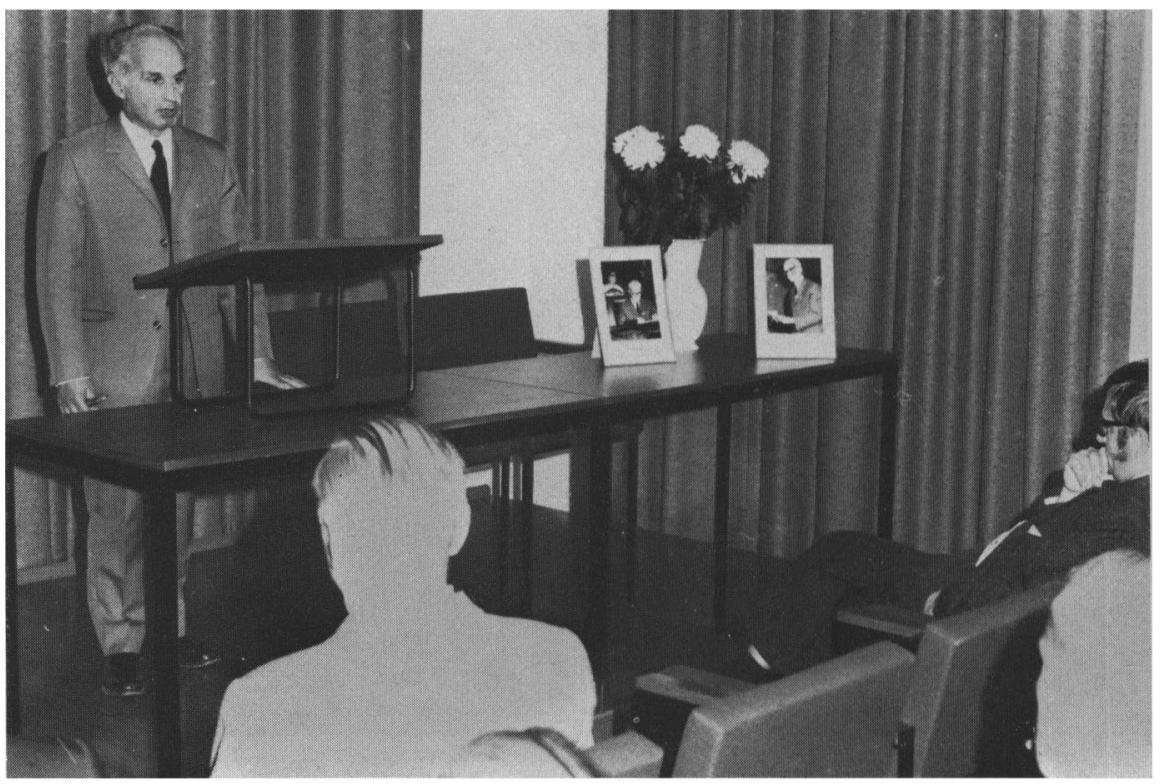

Plaut

Strohmeier

Kippenhahn

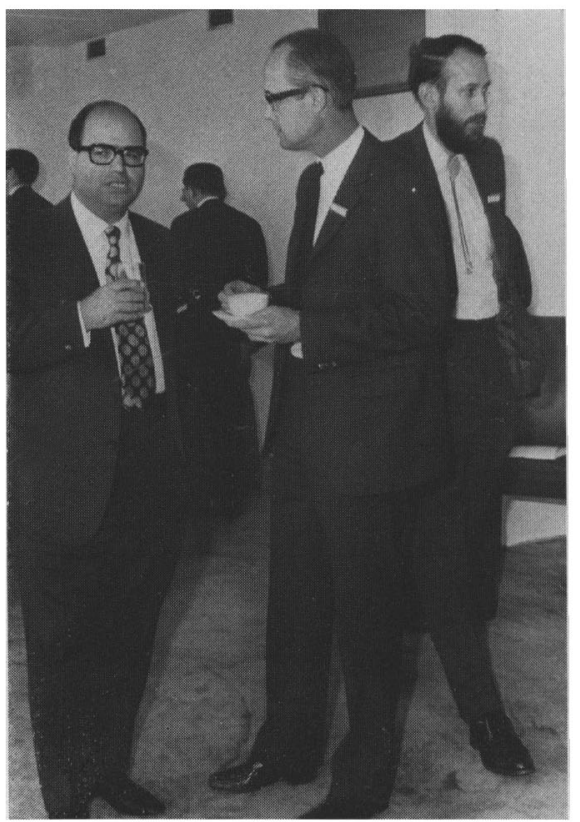

Mavridis
Vives Klinglesmith

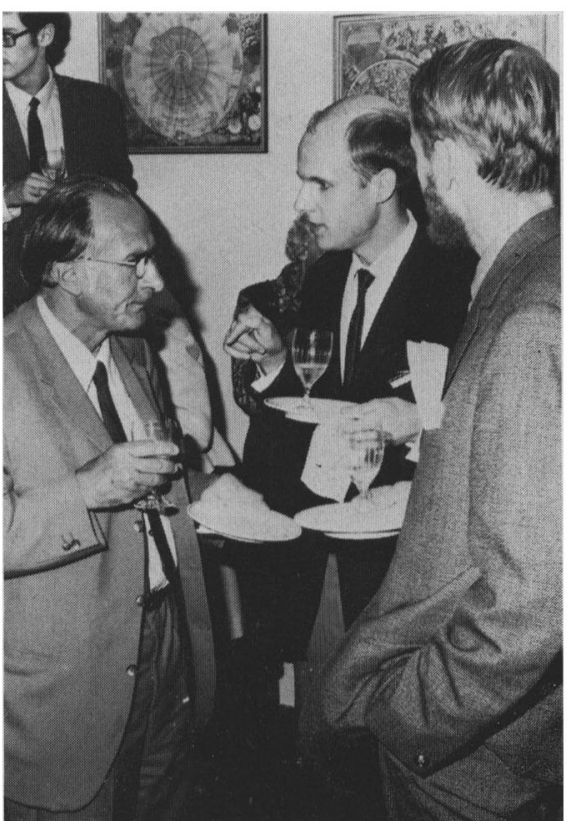

Behr

Ardeberg Klinglesmith 


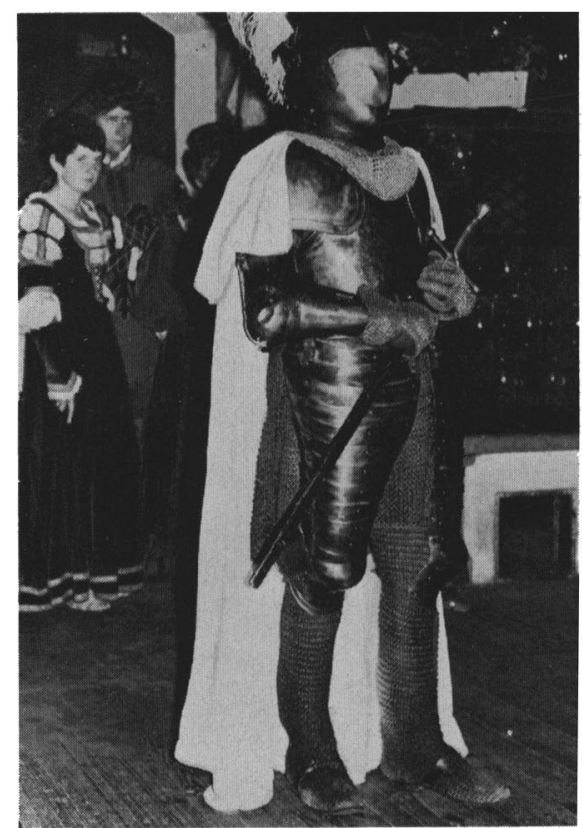

Ritter Kunibert

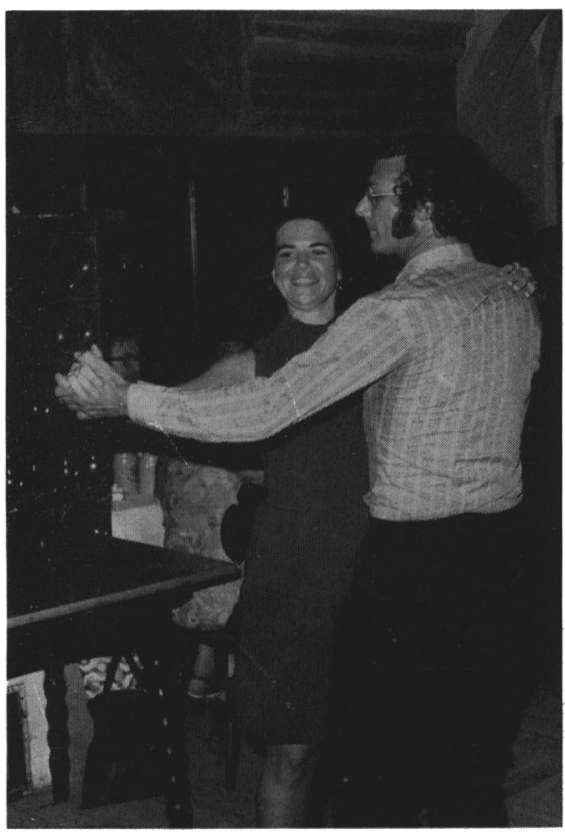

Perry

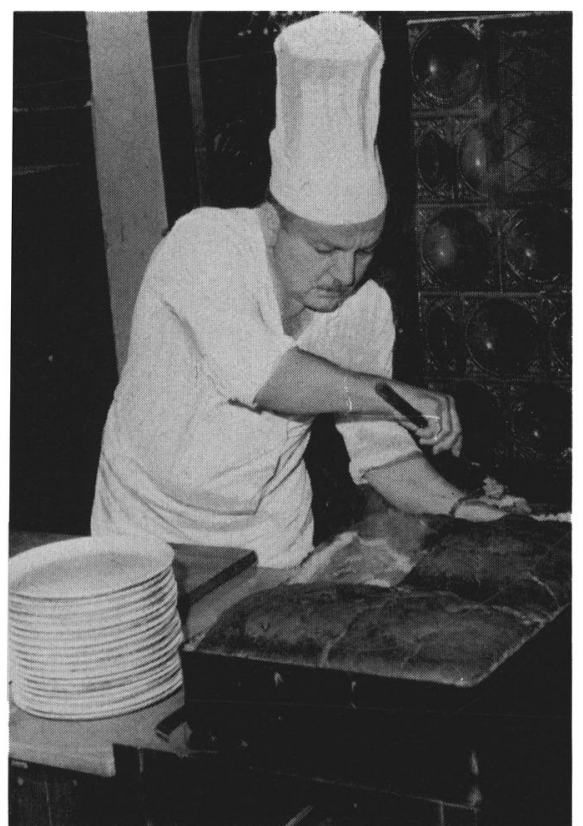

"Schinken im Brotteig“

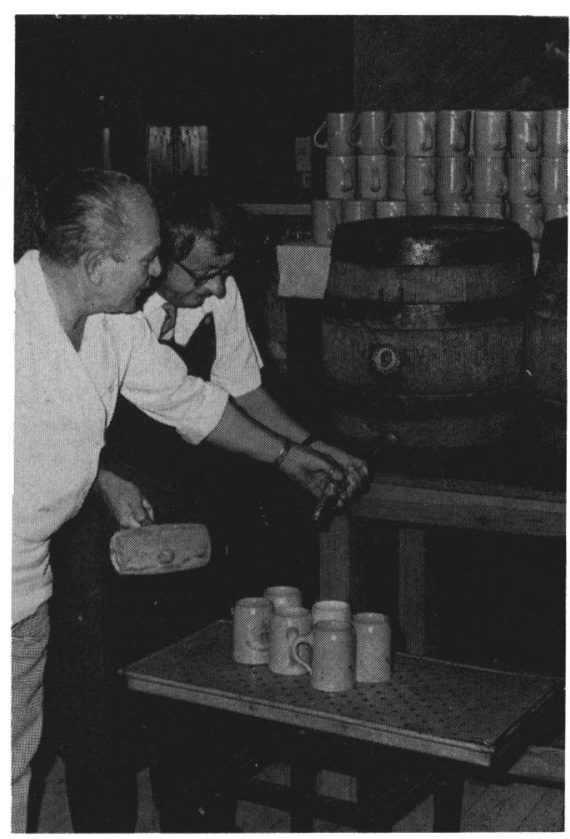

"Anzapfen“

All photographs by R. KNIGGE 


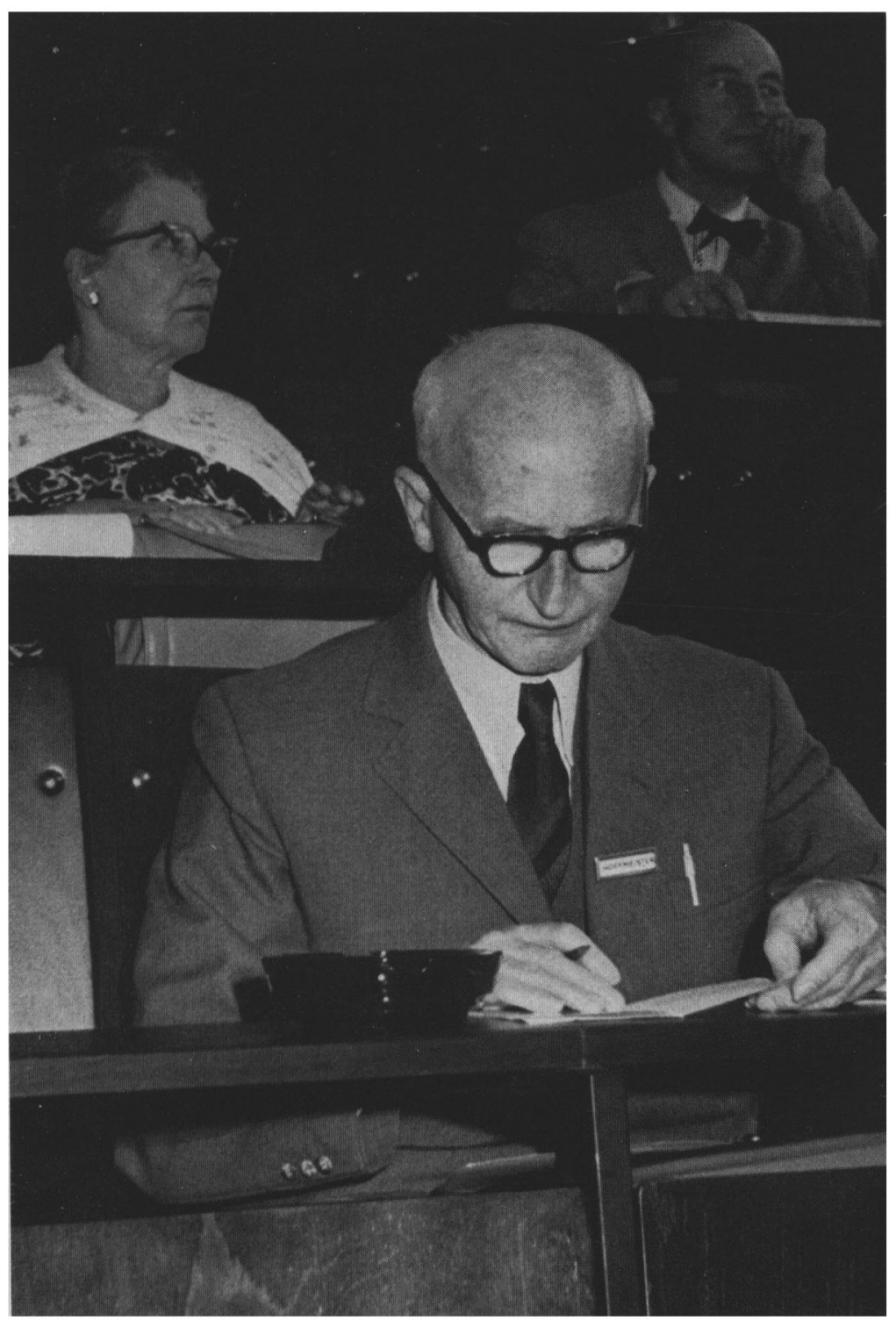

C. Hoffmeister t, IAU-Colloquium 1965, Bamberg 